\title{
Padrão de distribuição e estrutura populacional de Escallonia bifida Link \& Otto em área em vias de restauração no RS
}

\author{
Standard of distribution and population structure of escallonia bifida Link \& Otto in area in \\ restoration roads in $R S$
}

\author{
Roselene Marostega Felker \\ Ana Paula Moreira Rovedder \\ Maureen de Moraes Stefanello \\ Rafaela Badinelli Hummel \\ Bruna Balestrin Piaia \\ Aline Peccatti \\ Eliara Marin Piazza \\ Lucas Donato Toso \\ Betina Camargo
}

\section{Resumo}

Universidade de Santa Maria - UFSM - Santa Maria- Rio Grande do Sul - Brasil
A distribuição populacional das espécies florestais é um importante subsídio para o entendimento da estrutura da floresta, e seu manejo econômico e conservacionista. Desse modo, o objetivo do presente trabalho é o estudo da estrutura populacional da espécie nativa Escallonia bifida Link \& Otto, em área em vias de restauração na região central do RS. Foram amostrados todos os indivíduos da espécie presentes na classe regenerativa e arbórea nos anos de 2011 e 2012. Para avaliação da classe arbórea foram implantadas 16 parcelas de $10 \times 10 \mathrm{~m}$ (Classe III) e 64 subparcelas de $5 \times 5 \mathrm{~m}$ (Classe II) e para a regeneração 64 subparcelas de $5 \times 5 \mathrm{~m}$ (Classe I). Na classe III foram medidos indivíduos de CAP $\geq 15 \mathrm{~cm}$, na classe Il indivíduos de $5,1 \geq$ CAP $\leq 14,9 \mathrm{~cm}$ e classe I: $1 \leq \mathrm{CAP} \geq 5 \mathrm{~cm}$. Na avaliação da estrutura populacional foram analisados os parâmetros fitossociológicos, a distribuição espacial através do índice de Morisita e a distribuição diamétrica. A população de Escallonia bifida apresentou diminuição na frequência, densidade, valor de cobertura e importância de 2011 para 2012 e comportamento agregado. A distribuição diamétrica dos indivíduos apresentou modificações estruturais de 2011 para 2012, o que pode ser indicativo de mudanças sucessionais no fragmento em estudo.

\footnotetext{
Abstract

The population distribution of forest species is an important subsidy for the understanding of the structure of the forest, and its economic and conservationist management. Thus, the objective of the present work is the study of the population structure of the native species Escallonia bifida Link \& Otto, in an area undergoing restoration in the central region of RS. All individuals of the species present in the regenerative and arboreal class were sampled in the years 2011 and 2012. Six plots of $10 \times 10 \mathrm{~m}$ (Class III) and 64 subplots of $5 \times 5 \mathrm{~m}$ (Class II) were implanted to evaluate the tree class and to regenerate 64 subplots of $5 \times 5 \mathrm{~m}$ (Class I). Class III individuals were measured with CAP $\geq 15 \mathrm{~cm}$, in class II individuals of $5.1 \geq$ CAP $\leq 14.9 \mathrm{~cm}$ and class I: $1 \leq \mathrm{CAP} \geq 5 \mathrm{~cm}$. In the evaluation of the population structure were analyzed the phytosociological parameters, the spatial distribution through the Morisita index and the diametric distribution. The population of Escallonia bifida showed a decrease in frequency, density, coverage value and importance from 2011 to 2012 and aggregate behavior. The diameter distribution of individuals presented structural modifications from 2011 to 2012, which may be indicative of successional changes in the fragment under study
}

\section{Palavras-chave}

Escalloniaceae. Distribuição diamétrica. Índice de morisita..

\section{Keywords}

Escalloniaceae. Diametric distribution. Morisite index. 


\section{Introdução}

O manejo de florestas para exploração ou conservação, depende do entendimento das espécies e da sua dinâmica populacional. $O$ padrão de distribuição espacial permite conhecer os processos sucessionais e ecológicos que são fundamentais para a compreensão do ecossistema florestal, assim como para adotar medidas de manejo para conservação dos ambientes (RODE et al., 2010).

Conhecer a distribuição das espécies, pode ajudar a detectar padrões em comunidades vegetais e entender sua relação com os fatores que influenciam a estruturação populacional (FORTIN et al., 2002; GIEHL et al. 2007).

Segundo Silva et al. (2009) o estudo do padrão de distribuição espacial das espécies nativas é de extrema importância em estudos de ecologia, visto que, ajuda a entender como determinada espécie utiliza os recursos disponíveis e, ainda, porque é atribuído o sucesso do estabelecimento e da reprodução de determinadas espécies.

Escallonia bifida Link \& Otto, objeto desse estudo, é uma espécie pioneira de grande rusticidade, com grande potencial de uso em projetos de recuperação de áreas degradadas (FELKER et al., 2017). Possui ampla distribuição em áreas fragmentadas da Floresta Estacional, bioma Mata Atlântica, sendo considerada pelo Inventário Florestal contínuo do RS (2001), uma das cinco espécies mais abundantes em estágio iniciais da floresta estacional decidual e semidecidual.

No entanto, existe escassez de informações sobre a espécie, muito embora outras espécies do gênero, como E. pulverulenta, E. rubra e E. macrantha já são conhecidas e utilizadas em projetos ambientais e econômicos (CHALCOFF et al., 2006; MONTENEGRO et al., 2010), (VALDIVIA; NIEMEYER, 2006; SOUZA et al., 2007; FRITSCH, 2010).

Desse modo, o presente estudo, objetiva conhecer o padrão de distribuição e estrutura populacional da E. bifida, em fragmento florestal em vias de restauração, na região central do RS, e de posse destas informações contribuir para o conhecimento da espécie, estimulando sua utilização em estudos de restauração, recuperação e conservação ambiental.

\section{Material e métodos}

\section{Área de estudo}

O estudo foi realizado em fragmento de Floresta Estacional, localizado na unidade de conservação de proteção integral Parque Estadual Quarta Colônia, região central do RS. A área de estudo compreende um total de 5,7 hectares em processo natural de restauração, em diferentes estágios de sucessão. Anteriormente à instalação do parque, realizada em 2005, existiam propriedades rurais, áreas agrícolas e benfeitorias.

A região está em área de contato entre a encosta da Serra Geral e a Depressão Periférica, na zona limítrofe entre o Bioma Mata Atlântica e o Bioma Pampa. O relevo é forte ondulado, formado por morros e várzeas aluviais (STRECK et al., 2008).

O clima da região pode ser classificado como subtropical úmido, com precipitação média anual de $1708 \mathrm{~mm}$ e temperatura média anual de 19,2으 (MALUF, 2000).

\section{Amostragem de dados}

Foram amostradas três classes de distribuição populacional de E. bifida, incluindo a regeneração (Classe I) e estrato arbóreo (Classe II e III), em unidades amostrais distribuídas de forma aleatória (FELFILI et al., 2011). 
Para avaliação da classe arbórea foram implantadas 16 parcelas de 10 × $10 \mathrm{~m}$ (Classe III), com medição da circunferência à altura do peito (CAP) de todos os indivíduos no intervalo de: CAP $\geq 15 \mathrm{~cm}$ e 64 subparcelas de $5 \times 5 \mathrm{~m}$ para a classe II, com medição de todos os indivíduos no intervalo de: $5,1 \geq$ CAP $\leq 14,9 \mathrm{~cm}$.

Na regeneração (Classe I) foram alocadas 64 subparcelas de $5 \times 5 \mathrm{~m}$, com medição de todos os indivíduos da espécie presentes no intervalo de: $1 \leq \mathrm{CAP} \geq 5 \mathrm{~cm}$.

\section{Análise dos dados}

Foram determinados a frequência (FA) e densidade (DA) em valores absolutos e índice de cobertura e valor de importância para E. bifida em todas as classes de amostragem (MUELLER-DOMBOIS e ELLENBERG, 1974).

A distribuição espacial foi calculada pelo Índice de Morisita (IM) (MATTEUCCI e COLMA, 1982). O padrão espacial é considerado agregado quando o $\mid \mathrm{I}>1$. Como os dados não seguem distribuição normal foi aplicado o teste Qui-quadrado, para saber se o valor encontrado difere estatisticamente de 1, ao nível de 5\% de significância (BROWER e ZAR, 1984).

A distribuição diamétrica foi determinada de acordo com metodologia de Felfili e Rezende (2003), ajustada por meio da equação de Meyer, seguindo o modelo de floresta normal balanceada (SCHNEIDER, 2009).

A tabulação e o processamento dos dados e gráficos foram realizados por meio do programa MS Excel $2013^{\circ}$.

\section{Resultados e discussões}

\section{Estrutura populacional da E. bifida}

Foram amostrados 714 indivíduos de E. bifida, sendo 422 no período de 2011 e 292 em 2012. A espécie apresentou os maiores valores de densidade e frequência na classe arbórea (CAP $\geq 15 \mathrm{~cm}$ ), decrescendo para a regeneração, em ambos os períodos de avaliação. A espécie apresenta cerca de $80 \%$ de frequência na classe III e aproximadamente $50 \%$ na área amostral para a classe III e II, o que demonstra a grande colonização de E. bifida na área (Tabela 1). Sua alta densidade e frequência pode estar relacionada as condições do ambiente, áreas de capoeira e capoeirão que não limitam a entrada de luz.

Observa-se ainda que houve um decréscimo na população da espécie (FA, DA, IVI e IVC) de 2011 para 2012 (Tabela 1). As espécies diminuem sua densidade, à medida que se afastam das condições ótimas para seu desenvolvimento (MATTEUCCI e COLMA, 1982). O sombreamento ocasionado pelos indivíduos arbóreos de E. bifida podem ter contribuído para a diminuição na população da espécie, principalmente indivíduos regenerantes, visto que a espécie é exclusiva de ambientes heliófilos (VENZKE, 2012a). A espécie está presente nas fases iniciais de sucessão e coloniza ambientes quando ainda não existe um dossel formado (VENZKE, 2012a).

Em floresta estacional, no RS, em estágios de capoeira E. bifida substituiu a população de Bacharis sp. e proporcionou sombreamento do solo, resultando no surgimento de espécies de outros estágios sucessionais (VENZKE, 2012b). Segundo Venzke (2012b) nos estágios secundários a população da espécie fica restrita a indivíduos senis. 
Tabela 1: Parâmetros fitossociológicos da espécie E. bifida, em fragmento da Floresta Estacional Decidual, para o período de 2011 e 2012, nas classes de amostragem III: CAP $\geq 15$ $\mathrm{cm}$ ), II: $5.1 \geq \mathrm{CAP} \leq 14.9 \mathrm{~cm}$ e $\mathrm{l}: 1 \leq \mathrm{CAP} \geq 5 \mathrm{~cm}$

\begin{tabular}{c|c|c|c|c}
\hline $\mathbf{2 0 1 1}$ & FA & $\begin{array}{c}\text { DA } \\
\text { árv/há }\end{array}$ & IVI & IVC \\
\hline Classe III & 78,95 & 810,53 & 64,3 & 53,6 \\
Classe II & 59,37 & 1093,7 & 67,74 & 54,0 \\
Classe I & 39,06 & 450,0 & - & - \\
\hline $\mathbf{2 0 1 2}$ & FA & DA & IVI & IVC \\
& & árv/há & & 53,51 \\
Classe III & 78,95 & 705,26 & 63,79 & 36,08 \\
Classe II & 45,31 & 637,5 & 47,59 & - \\
Classe I & 32,81 & 350,0 & - & \\
\hline
\end{tabular}

FA (Frequência absoluta em \%); DA (Densidade absoluta, árvores/ha); IVI (Índice de valor de importância); IVC (Índice de valor de cobertura);

\section{Distribuição espacial}

Os valores para o Índice de Morisita em ambos os períodos de avaliação (Tabela 2), revelaram comportamento agregado para a espécie. Observa-se ainda, que o padrão de agregação aumenta do estrato arbóreo em direção à regeneração e do período de 2011 para 2012. Possivelmente os indivíduos da espécie estão encontrando menores aberturas de dossel para desenvolvimento e, consequentemente, acabam se aglomerando em áreas de maior intensidade luminosa. $O$ padrão espacial das árvores em uma floresta é altamente influenciado por variáveis abióticas dentre estas, uma das principais variáveis está a disponibilidade de luz (SANTANA et al. 2011).

Gabriel et al. (2006) comparando o padrão espacial de diferentes categorias de tamanho de Cupania vernalis também verificou que os indivíduos regenerantes estavam mais agrupados, sendo a luminosidade o fator responsável pelo agrupamento dos indivíduos jovens.

Segundo Kanieski et al. (2012) o padrão agregado sugere que a espécie está se desenvolvendo nos mesmos locais dentro da área de estudo, seguindo as exigências da espécie nos sítios, que neste caso, segundo as características ecológicas da E. bifida, pode ser a presença de luminosidade. Segundo Felker et al. (2017) a luz é um fator importante no desenvolvimento de E. bifida, podendo limitar o desenvolvimento da espécie em locais com baixa luminosidade.

Espécies de estágios iniciais de sucessão e que habitam locais alterados como clareiras e bordas de florestas, geralmente apresentam tendência à distribuição agregada (NASCIMENTO et al., 2001). 
Tabela 2. Distribuição espacial da espécie E. bifida, obtida através do Índice de Morisita, para fragmento de Floresta Estacional Decidual, RS

\begin{tabular}{c|c|c}
\hline \multicolumn{3}{c}{ Índice de Morisita* } \\
\hline Classes de amostragem & 2011 & 2012 \\
\hline Classe III (CAP $\geq 15 \mathrm{~cm})$ & $1,8^{*}$ & 1,9 \\
Classe II $(5,1 \leq$ CAP $\geq 14,9 \mathrm{~cm})$ & 2,7 & 3,4 \\
Classe I $(1 \leq$ CAP $\geq 5 \mathrm{~cm})$ & 2,8 & 4,1 \\
\end{tabular}

*valores diferem estatisticamente de 1, ao nível de 5\% de significância, pelo teste do Qui-quadrado

\section{Distribuição diamétrica}

Observou-se variações na estrutura diamétrica do ano de 2011 para 2012 (Figura 1 e Figura 2). No ano de 2011 percebe-se que a distribuição está próxima ao normal, com tendência ao "J-invertido", com maiores proporções de indivíduos nas classes de menores circunferência a altura do peito (CAP), indicando a capacidade regenerativa da espécie na floresta (Figura 1).

No entanto no período de 2012, a distribuição diamétrica da E. bifida, apresenta-se descontínua, mostrando que existe um desbalanceamento diamétrico dos indivíduos em determinadas classes, neste caso na classe da regeneração (CAP de 1 a $5 \mathrm{~cm}$ ) (Figura 2).

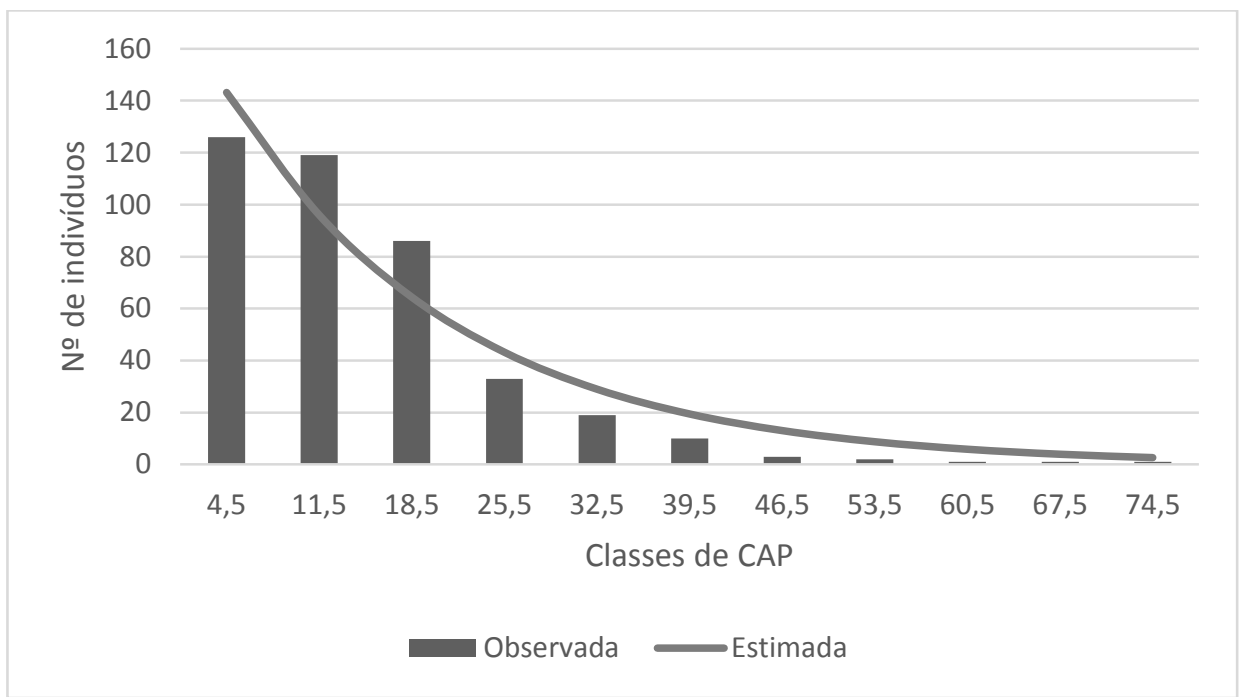

Figura 1. Distribuição diamétrica observada e estimada da E. bifida em Fragmento de Floresta Estacional Decidual, baseada na Circunferência à altura do peito (CAP), para o ano de 2011, ajustada pelo equação de Meyer, $R^{2}$ : 0,7625, erro: 0,4. 


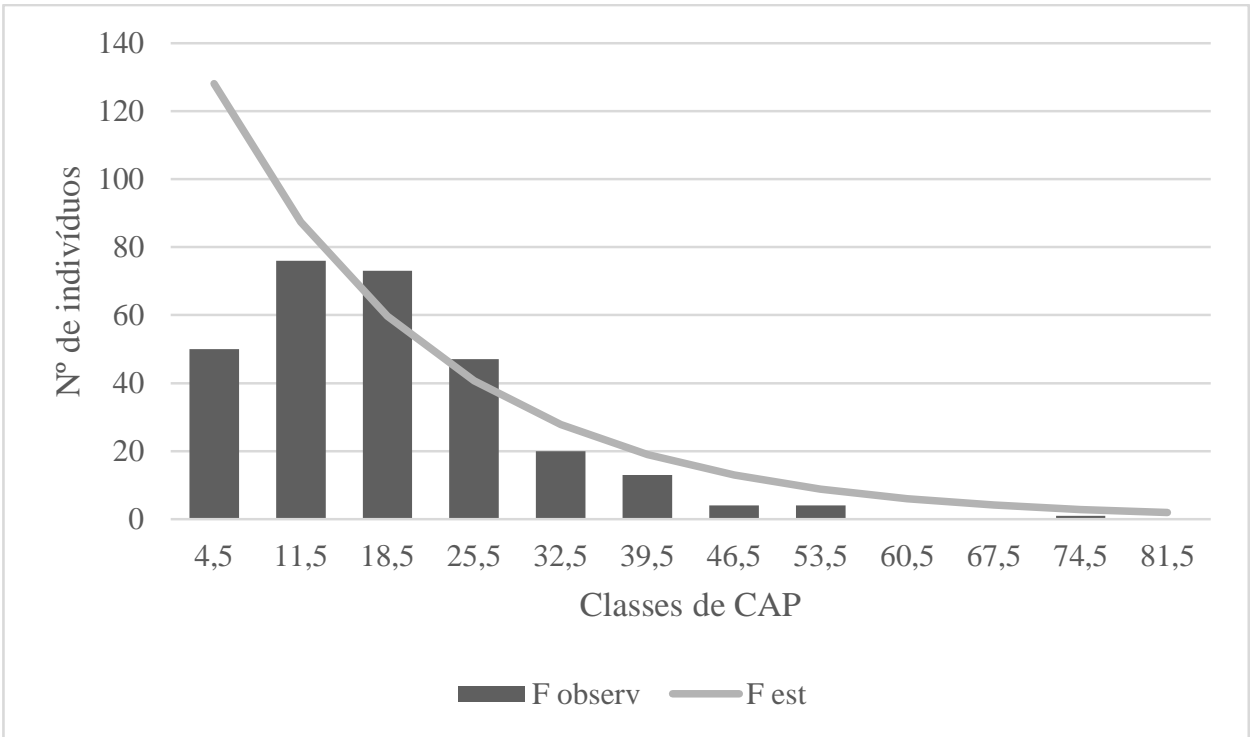

Figura 2. Distribuição diamétrica observada e estimada da E. bifida em Fragmento de Floresta Estacional Decidual, baseada na Circunferência à altura do peito (CAP), para o ano de 2012, ajustada pela equação de Meyer, $R^{2}: 0,7753$, erro: 1,21

De acordo com Souza et al. (2012) a estrutura do tipo "J-invertido", com muitos indivíduos concentrados nas menores classes de tamanho e poucos indivíduos nas maiores classes, sugere que as populações que compõem uma comunidade são estáveis e autorregenerativas e que existe um balanço entre mortalidade e o recrutamento dos indivíduos.

Segundo Silva Júnior e Silva (1988), os histogramas de frequência diamétrica podem retratar os acontecimentos pretéritos e futuros de determinada floresta. Nesse caso supõe-se que o número de indivíduos presente na regeneração está diminuindo, devido a fatores limitantes, que nesse caso poderá ser o fator luminosidade. Considerando a sucessão ecológica, a diminuição dos indivíduos regenerantes seria um fator natural. Poucos indivíduos jovens podem indicar o declínio da população no ambiente (CONDIT et al. 1998).

\section{Conclusão}

A população de E. bifida está se modificando ao longo do tempo, e por se tratar de espécie pioneira, exigente em luz, conclui-se que a tendência natural será a saída da espécie om o avançar da sucessão ecológica.

A estrutura e distribuição de E. bifida pressupõe potencial desta para colonização de áreas abertas, e facilitadora para o aporte e desenvolvimento de espécies tolerantes à sombra, podendo ser estudada como espécie chave em projetos de recuperação e restauração locais.

\section{Agradecimentos}

Os autores agradecem o Conselho Nacional de Desenvolvimento Científico e tecnológico (CNPq), pelo auxílio financeiro na execução do estudo.

\section{Referências}

1. BROWER, J. E; ZAR, J.H. 1984. Field e laboratory methods for generalecology. 2 ed.

Dubuque: Wm.C.Brow Publ. 226 p.

Cadernos de Pesquisa [ISSN 1677-5600]. Santa Cruz do Sul, v. 29, n. 2, p. 16-24, mai./ago. 2017

https://online.unisc.br/seer/index.php/cadpesquisa/index 
CHALCOFF V. R.; AIZEN M.A.; GALETTO L. Nectar concentration and composition of 26 species from the temperate forest of South America. Annals of Botany, Oxford, v.97, n. 3, p.413-421, 2006. CONDIT, R.; SUKUMAR, R.; HUBBELL, S.; FOSTER, B. Predicting population trends from size distributions: a direct test in a tropical tree community. The American Naturalist, Chicago, v. 152, p. 495-509, 1998.

FELFILI, J. M.; REZENDE, R. P. 2003. Conceitos e métodos em fitossociologia. Brasília: Ed. UNB, $68 \mathrm{p}$.

FELFILI, J. M.; ROITMAN, I.; MEDEIROS, M.M.; SANCHEZ, M. Procedimentos e métodos de amostragem de vegetação. In: FELFILI, J. M.; EISENLOHR, P.V.; MELO, M.M.R.F.; ANDRADE, L.A.; MEIRA-NETO, J.A.A. (Org.). (Org.). Fitossociologia no Brasil: métodos e estudos de casos. Viçosa, MG: Universidade Federal de Viçosa, 2011. p. 86-121. FELKER, R. M.; ROVEDdeR, A. M.; STEFANELLO, M. M.; HUMMEL, R. B.; PIAIA, B. B.; CAMARGO, B. Distribuição de Escallonia bifida Link \& Otto em relação à variáveis ambientais, em fragmento florestal em vias de restauração no RS. Nativa, v.5, n.2, p.133-137, 2017.

7. FORTIN, M. J.; DALE, M. R. T. \& HOEF, J. Spatial analysis in ecology. In: A. H. ElSHAARAWI \& W.W. PIEGORSCH (eds.). Encyclopedia of Environmetrics, 2002, p. 20512058.

FRITSCH, M. Estudo taxonômico do gênero Escallonia mutis ex I.f. (Escalloniaceae) no estado do Paraná, Brasil. 2010. 101f. Dissertação (Mestrado em Ciências Biológicas) Universidade Federal do Paraná, 2010.

9. GABRIEL, C.; AZAMBUJA, B.; GIEHL, E.L.H.; EISINGER, S.M. 2006. Estrutura populacional de Cupania vernalis Camb. em uma floresta estacional no sul do Brasil. In: Distribuição espacial de espécies arbóreas em uma floresta estacional. Anais do LVII Congresso Nacional de Botânica. Gramado, Universidade Federal do Rio Grande do Sul. em uma floresta estacional em Santa Maria, Sul do Brasil. Pesquisas, botânica, $n^{\circ} 58$ : 215-226, 2007. KANIESKI, M. R.; LONGHI, S. J.; NARVAES, I, S.; SOARES, P. R. C.; LONGHI-SANTOS, T.; CALLEGARO, R. M. Diversidade e padrões de distribuição espacial de espécies no estágio de regeneração natural em São Francisco de Paula, RS, Brasil. Floresta, v. 42, n. 3, 2012. p. $509-518$. 
MALUF, J. R. T. Nova classificação climática do estado do Estado do Rio Grande do Sul. Revista Brasileira de Agrometeorologia, v. 8, n. 1, 2000. p. 141-150.

MATTEUCCI, S. D.; COLMA, A. 1982. Metodologia para el estudio de la vegetacion. Washington: Secretaria General de la Organizacion de los Estados Americanos Programa Regional de Desarrollo y Tecnologico. 169 p.

MONTENEGRO, G.; PEÑA, R. C.; PIZARRO, R. Multivariate analysis of pollen frequency of the native species Escallonia pulverulenta (Saxifragaceae) in Chilean honeys. Revista Brasileira de Botânica, v. 33, n. 4, p. 615-630, 2010.

MUELLER-DOMBOIS, D.; H. ELLENBERG. 1974. Aims and Methods of Vegetation Ecology. $547 \mathrm{p}$.

NASCIMENTO, A. T.; LONGHI, S. J.; BRENA, D. A. Estrutura e padrões de distribuição espacial de espécies arbóreas em uma amostra de floresta Ombrófila Mista em Nova Prata, RS. Ciência Florestal, v.11, n.1. p. 105-119, 2001.

RIO GRANDE DO SUL. Inventário Florestal Contínuo do Rio Grande do Sul. Porto Alegre: FATEC/SEMA, 2001. Disponível em: <www.ufsm.br/ifcrs>. Acesso em: 17 ago. /2013.

SANTANA, J. A. S.; VIEIRA, F. De A.; PACHECO, M. V.; DE OlIVEIRA, P. R. S. Padrão de distribuição e estrutura diamétrica de Caesalpinia pyramidalis Tul. (Catingueira) na Caatinga do Seridó. Revista de Biologia e Ciências da terra, v.11, n.1, p. 116-122, 2011.

SCHNEIDER, P. R.; SCHNEIDER, P. S. P.; SOUZA, C. A. M. 2009. Análise de Regressão aplicada à Engenharia Florestal. 2. Ed- Santa Maria: FACOS. 294 p.

SILVA JÚNIOR, M. C.; SILVA, A. F. Distribuição dos diâmetros dos troncos das espécies mais importantes do cerrado na Estação Florestal Experimental de Paraopeba (EFLEX) MG. Acta Botanica Brasilica, v. 2, n.1, p. 107-126, 1988.

SILVA, B. M.; ROSSI, A. A. B.; ENCINAS, J. F.; CARVALHO, M. L. DA S.; DA SILVA, C. J. Estrutura e padrões de distribuição espacial de duas espécies de Theobroma em um parque de preservação permanente no norte do estado de Mato Grosso. Enciclopédia biosfera, v.9, n.17; p. 2013.

SILVA, K. E.; MARTINS, S. V.; SANTOS, N. T.; RIBEIRO, C. A. A. S. Padrão espacial de espécies arbóreas tropicais. In: MARTINS, S. V (Ed). Ecologia de Florestas Tropicais. Viçosa: Ed. UFV, 2009. p. 216 - 241.

SOUZA, L. P.; ANGELO, A. C.; CURCIO, G. R.; BONNET, A.; GALVÃO, F. Recuperação ambiental em área de estepe do Primeiro Planalto Paranaense, mediante plantio de espécies arbóreas. Notas Científicas. Pesquisa Florestal brasileira, Colombo, n. 55, p. 95 $101,2007$.

Cadernos de Pesquisa [ISSN 1677-5600]. Santa Cruz do Sul, v. 29, n. 2, p. 16-24, mai./ago. 2017 https://online.unisc.br/seer/index.php/cadpesquisa/index 
24. SOUZA, P. B.; DE SOUZA, A. L.; ALVES MEIRA NETO, J. A. Estrutura diamétrica dos estratos e grupos ecológicos de uma área de Floresta Estacional Semidecidual,em Dionísio, MG. Rev. Árvore vol.36 no.1, 2012.

25. STRECK, E. V.; KÄMPF, N.; DALMOLIN, R. S. D.; KLAMT, E.; NASCIMENTO, P. C.; GIASSON, E. \& PINTO, L.F.S. 2008. Solos do Rio Grande do Sul. 2 ed. Porto Alegre: EMATER/RS. 222 p.

26. VALDIVIA C. E.; NIEMEYER, H. M. Do pollinators simultaneously select for inflorescence size and amount of floral scents? An experimental assessment on Escallonia myrtoidea. Austral Ecology, Alice Springs, v. 31, n. 7, p. 897-903, 2006.

27. VENZKE, T. S. Florística de comunidades arbóreas no Município de Pelotas, Rio Grande do Sul. Rodriguésia (Online), v. 63, p. 571-578, 2012.

28. VENZKE, T. S. Florística, estrutura e síndrome de dispersão de sementes em estágios sucessionais de mata ciliar no município de Arroio do Padre, RS, Brasil. 2012. $75 f$. Dissertação de mestrado. (Mestrado em Botânica) - Universidade Federal de Viçosa, MG, 2012. 